Z. klin. Chem. u. klin. Biochem.

9. Jg., S. 53-56, Januar 1971

\title{
Kombinierte elektrophoretische Darstellung von Proteinen und Kohlenhydraten auf einem Streifen
}

\author{
Von J. Barrollrer und V. Busse \\ Aus dem Pbysiologisch-Chemiscben Institut und der Cbirurgiscben Universitätsklinik \\ - Klinikum Westend - der Freien Universität Berlin
}

(Eingegangen am 13. August 1970)

\begin{abstract}
Es wird eine Methode beschrieben, Proteine und Glykoproteine nach clektrophoretischer Auftrennung auf einem Streifen anzufärben. Mit Tartrazin für die Proteinfärbung und rosanilinschwefliger Säure für die Kohlenhydratfärbung wurden Farbstoffe gewählt, deren Absorptionsmaxima so weit auseinander liegen, daß bei der Messung des einen die Absorption des anderen Farbstoffs praktisch zu vernachlässigen war. Eine exakte Zuordnung dieser beiden Serumbestandteilc zueinander ist so möglich. Bei der Anwendung der Methode im klinisch-chemischen Laboratorium konnten Besonderheiten im Verhalten von Proteinen zu Glykoproteinen bei Paraproteinämien (Plasmocytom und M. WALDENSTRÖM) aufgezeigt werden.
\end{abstract}

\section{The combined electrophoretic separation of proteins and carbobydrates on one strip}

A method is described for the location of both proteins and glycoproteins on the same electrophoresis strip. Using tartrazine for the protein and rosaniline-sulphurous acid for the carbohydrate, colours are obtained with widely different absorption maxima, so that each may be measured with practically no interference by the other. Each serum component can therefore be exactly identified. In the clinical chemical laboratory, the method revealed peculiarities in the ratio of proteins to glycoproteins in paraproteinemias (plasmacytoma and M. WALDENSTRÖM).

Seit den Untersuchungen von MörNER (1) konnte gezeigt werden, daß Kohlenhydrate Bestandteil der Serumproteine sind. Im folgenden wurden dann diese Kohlenhydrate in den Serumfraktionen von Rimington (2) und SöRENSEN (3) durch Fällungsmethoden analysiert. Die ersten elektrophoretischen Untersuchungsmethoden stammen von Tiselius (4), Körw und Grönvali (5) und Wunderly und Piller (6).

Es handelt sich dabei um jene eiweißgebundenen Kohlenhydrate, die im Unterschied zu den Mukopolysacchariden bei der sauren Eiweißfällung mit Trichloressigsäure ausfallen, also die eigentlichen Glykoproteine. Sie enthalten nur $1-5 \%$ Zucker in Form von Mannose, Hexosamin, Galaktose und wechselnden Mengen von Sialinsäure und Neuraminsäure.

Eine genaue quantitative Aussage über die Beziehung Glykoprotein-Serumprotein ist durch den stark wechselnden Anteil verschiedener Zucker mit ihren unterschiedlichen Farbstoffbindungen nicht möglich. Relative Unterschiede der Kohlenhydrat-Mengen in den Eiweißfraktionen lassen sich aber sehr gut erkennen. In dieser Arbeit soll erstmals eine Methode beschrieben werden, die eine einwandfreie Bestimmung von Kohlenhydraten und Proteinen auf einem Elektrophoresestreifen ermöglicht. Bei den bisher geübten Verfahren (Wunderly und Mitarbeiter) (6) war die gemeinsame Bestimmung von Kohlenhydraten und Proteinen nur auf mehreren Streifen durchführbar. Auf diese Weise ist eine exakte Zuordnung von Protein- und Kohlenhydrat-Zonen zueinander schwierig, da es kaum gelingt, eine völlig gleiche Wanderung auf parallelen Streifen zu erzielen. Das Prinzip der Methode besteht darin, für die Proteinfärbung einen Farbstoff zu wählen, dessen Absorptionsmaximum in einem Wellenlängenbereich liegt, in dem die Absorption der Kohlenhydrat-Färbung praktisch zu vernachlässigen ist (s. Abb. 1). Man mißt dann die Proteinfärbung bei einer Wellenlänge, bei der die Kohlenhydrat-Färbung noch keine Absorption zeigt und umgekehrt.

Bei der Ủberprüfung einiger Anwendungsbeispiele mit dieser Methode fielen bestimmte Eigentümlichkeiten im Verhalten der Kohlenhydrate bei Paraproteinämien auf, so daß hierauf kurz eingegangen wird.

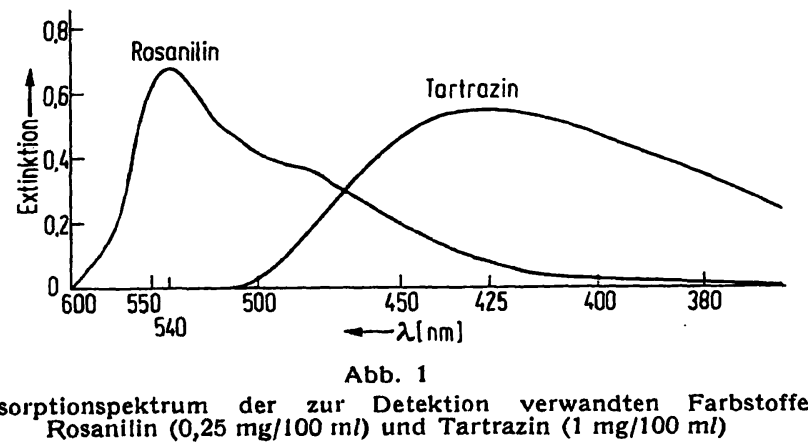

\section{Material und Methodik}

Reagenzien

1. Perjodsäure 1proz. in dest. Wasser frisch ansetzen,

2. Rosanilinschweflige Säure: $0,5 \mathrm{~g}$ Rosanilin in $100 \mathrm{~m} /$ heißcm dest. Wasser lösen. Nach Abkühlen auf ctwa $50^{\circ} 1 \mathrm{~g}$ Natriumhydrogensulfit und $36 \mathrm{~m} / 1 \mathrm{~N}$ HCL hinzugeben. Dieses Gemisch 
über Nacht stehen lassen, bis es farblos bzw. leicht bräunlich ist. Dic Lösung wird farblos gemacht, indem man 0,5-1 g Aktivkohlc hinzugibt, umschwenkt und nach $30 \mathrm{Min}$. abfiltriert. (Diese Lösung ist im Kühlschrank unbeschränkt haltbar!),

3. Bisulfitwasser: $10 \mathrm{~g}$ Natriumhydrogensulfit $+1 l$ dest. Wasser $+6 \mathrm{ml}$ Eisessig,

4. Tartrazin: 0,1proz. in Methanol/0,2N Oxalsaure 1:2(v/v),

5. Transparenzbad: $100 \mathrm{ml}$ Äthanol $+25 \mathrm{ml}$ Eisessig.

Geräte

Elektrophoresekammer (Shandon), Spektralphotometer mit Densitogrammzusatz und Kompensations-Schreiber (C. Zeiss).

\section{Durchführung}

Die Serumelektrophorese wird bei $\mathrm{pH} 8,8$ wie in einer früheren Mitteilung beschrieben (7), ausgeführt. Die denaturierten, noch ungefärbten Streifen werden in Benzol, Toluol oder Benzin kurz eingetaucht, um Lipide und freie Fettsäuren in Lösung gehen zu lassen, weil ungesättigte Fettsäuren bzw. Lipoide nämlich auch eine PAS-Färbung geben.

Anschließend nach Verdampfen der Flüssigkeit $45 \mathrm{Min}$. langes $\mathrm{Bad}$ in 1proz. Perjodsäure, $10 \mathrm{Min}$. Auswaschen unter fließendem Leitungswasser. Danach $45 \mathrm{Min}$. in Rosanilinschwefliger Säure färben, $6 \times 10 \mathrm{Min}$. in Bisulfitwasser waschen und nochmals $60 \mathrm{Min}$. unter fließendem Leitungswasser wässern. Im Anschluß hieran erfolgt die Proteinfärbung. Der kohlenhydratgefärbte Streifen wird $15 \mathrm{Min}$. in 0,1proz. Tartrazinlösung gefärbt, Auswaschen des überschüssigen Farbstoffes in Methanol/Oxalsäure 1:2 (v/v) bis zur Entfärbung des Streifens. Der Streifen kommt kurz in Methanol und wird dann auf eine entsprechende Glasplatte $(150 \times 30 \mathrm{~mm})$ aufgezogen. Die Transparenz erfolgt nach Transparenzbad von $15-20$ Sek. im Trockenschrank bei $60^{\circ}$ nach etwa $30 \mathrm{Min}$.

Anfang und Ende des Elektropherogramms werden mit Markierungsstrichen gekennzeichnet. Im Densitometriezusatz des Spektralphotometers wird zunächst das Densitogramm des Proteinelektropherogramms bei $425 \mathrm{~nm}$ ermittelt und nach Drehung des Streifens um $180^{\circ}$ erfolgt die spiegelbildliche Aufzeichnung des Densitogramms des Kohlenhydrat-Elektropherogramms bei $540 \mathrm{~nm}$. Protein- und Kohlenhydrat-Aufzeichnung wurden immer bei gleichbleibender Transportgeschwindigkeit von Schreiber und Transportschlitten und derselben Empfindlichkeitsstufe des Photometers vorgenommen.

\section{Ergebnisse}

Seren von 25 gesunden Menschen dienten uns zur Untersuchung der Kohlenhydrate und Proteine mit der Serumelektrophorese. Die gefärbten Streifen bieten nebeneinander die mit dem Auge nur schwach zu erkennenden gelb gefärbten Proteinfraktionen und die deutlich rot-violett gefärbten Kohlenhydrat-Fraktionen. Die photographische Wiedergabe ist aus diesen Gründen unzweckmäßig. Die Dokumentation gelingt nur mittels eines Densitogramms.

Abbildung 2 zeigt das typische Verhalten aufgetrennter Kohlenhydrate und Proteine gesunder Normalpersonen. Erwartungsgemäß finden wir den Hauptanteil der Kohlenhydrate in der Region der $\alpha$ - und $\beta$-Globuline. Regelmäßig lassen sich fünf Kohlenhydrat-Fraktionen darstellen. Es besteht eine exakte Zugehörigkeit der einzelnen Kohlenhydrat-Fraktionen zu den bekannten Erhebungen im Proteinelektropherogramm. So lassen sich jeweil im Albumin-, $\alpha_{1^{-}}, \alpha_{2^{-}}, \beta$ - und $\gamma$-GlobulinBereich voneinander getrennte korrespondierende Koh-
lenhydrat-Gipfel nachweisen, die im folgenden mit den römischen Ziffern I (Albumin) -V ( $\gamma$-Globulin) gekennzeichnet werden sollen. Die höchste Erhebung im Kohlenhydrat-Bereich finden wir regelmäßig im Gipfel III (entspricht $\alpha_{2}$-Globulin). Auffallend war bei den untersuchten Seren der relativ hohe Ausschlag in I und eine versetzte Erhebung (V) im $\gamma$-Globulin-Bereich. Hier fallen die Gipfelpunkte der Kohlenhydrate und der Proteine nicht genau zusammen.

Die Untersuchung von Seren bei Lebercirrhose (Abb. 3) ergibt in bezug auf die Kohlenhydrate einen dem Proteinelektropherogramm ähnlichen stumpfbogigen Gipfel bzw. ein Plateau in V, das gegenüber dem normalen Vergleichkollektiv deutlich vergrößert ist. Die übrigen Kohlenhydrat-Anteile zeigen in Übereinstimmung die relativen Veränderungen der Serumproteine.

Die Abbildungen 4 und 5 zeigen je ein kombiniertes Kohlenhydrat- und Proteinelektropherogramm bei Plasmocytom und M. WALDENSTröm. Auffälliger Unterschied ist die schmalbasige, deutlich überhöhte Kohlenhydrat-Zacke beim M. WALDENSTRöm in V, die bei gleichbleibender Empfindlichkeit des Photometers den Proteingipfel im $\gamma$-Globulin-Bereich überragt. Außerdem ist diese Zacke auf den $\gamma$-Globulin-Bereich beschränkt. Beim Plasmocytom ist je nach immunologischer Differenzierung der Kohlenhydrat-Gipfel unterschiedlich im Globulin-Bereich lokalisiert (Abb. 6) und erreicht zumindest im $\gamma$-Globulin-Bereich nicht die Höhe des zugehörigen Proteingipfels (Abb. 4 u. 6).

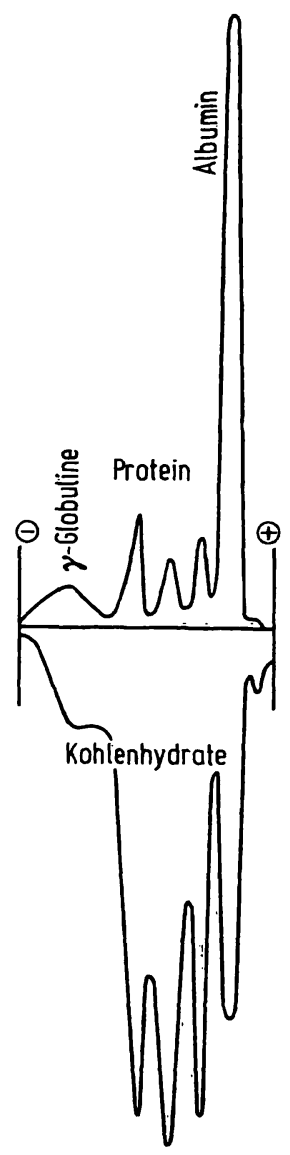

Abb. 2

Protein- und Kohlenhydrat-Densitogramm eines Normalserums

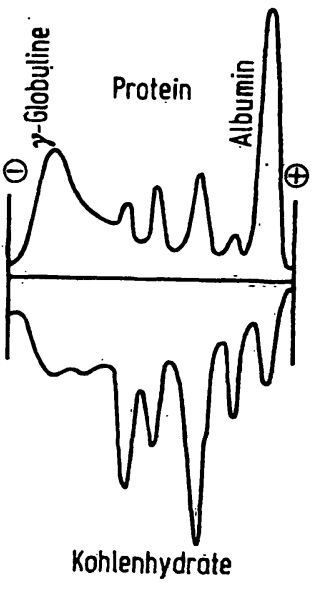

Abb. 3

Protein- und Kohlenhydrat-Densitogramm eines Serums bei Lebercirrhose 


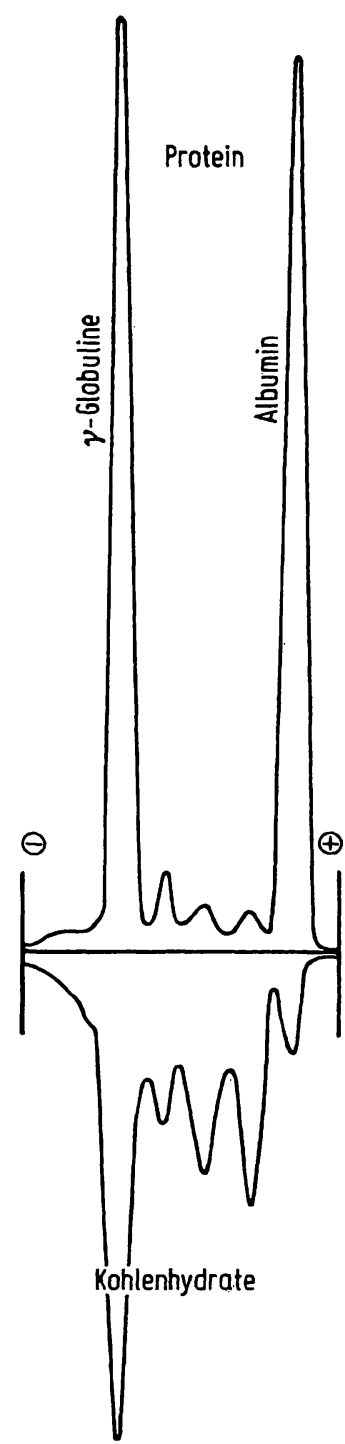

Abb. 4

Protein- und KohlenhydratDensitogramm eines Serums bei Plasmocytom (vgl. auch Abb. 6)

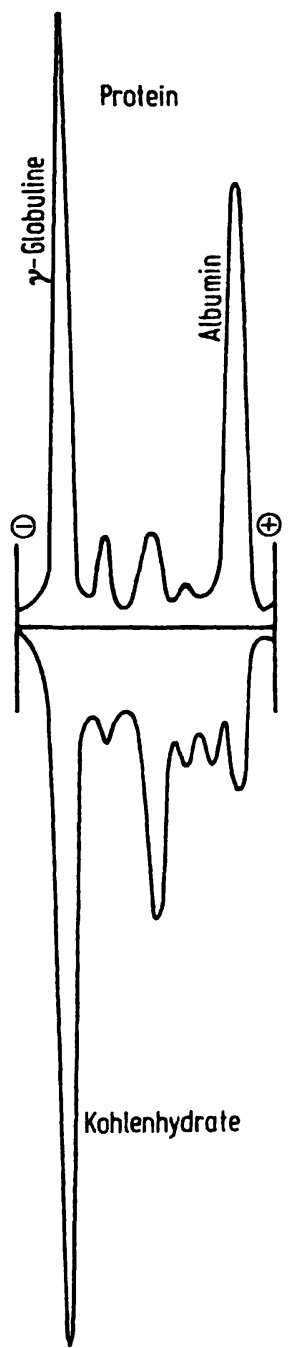

Abb. 5

Protein- und KohlenhydratDensitogramm eines Serums bei M. WALDENSTRÖM

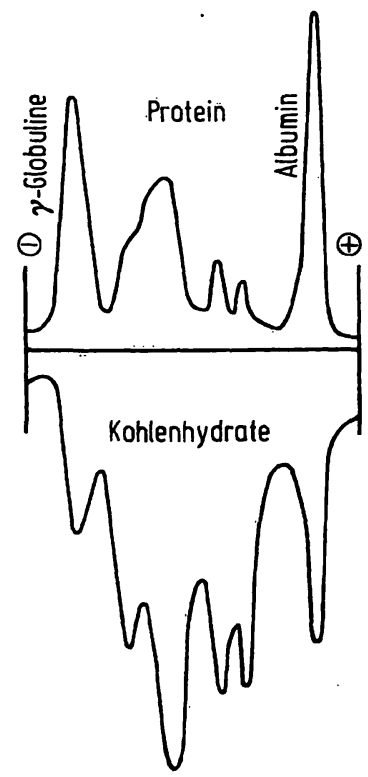

Abb. 6

Protein und Kohlenhydrat-Densitogramm eines Serums bei Plasmocytom (vgl. atch Abb: 4)

\section{Diskussion}

Dic hier mitgeteilten Kohlenhydrat-Elektropherogramme von Normalseren sind im Vergleich zu Untersuchungen anderer Autoren (INFANTE (8), WUNDERLY (6), BötTIGer (9), Scheiffarth (10)) in guten Einklang zu bringen. Ubereinstimmend kann festgestellt werden, daß normalerweise im $\alpha_{2}$ - und $\beta$-Globulin-Bereich der Kohlenhydrat-Anteil (II) am größten ist. Die Angaben über den Anteil (I) der Glykoproteine im AlbuminBereich sind sehr unterschiedlich $(0,76-15 \%)$, während die relative Verteilung der anderen Fraktionen (II-IV) von allen Autoren etwa gleichsinnig angegeben werden. Quantitative Angaben werden nur dann gemacht, wenn durch chemische Hilfsmethoden die einzelnen Kohlenhydrat-Komponenten exakt bestimmt werden können. Der hohe Kohlenhydrat-Anteil in I unserer Diagramme ist zu einem beträchtlichen Teil durch die Trübung von Licht durch gefärbte Albuminmoleküle bedingt. Beim Anfärben gehen die zunächst gleichmäßig in der elektrophoretischen Zone verteilten Proteine in gefärbte Körnchen über, da alle sauren Wollfarbstoffe (Tartrazin, Neucoccin, Pouceau S) Eiweißfällungsmittel sind. Eine Beeinflussung der Kohlenhydrat-Färbung bei der Wellenlänge für die Proteinmessung $(425 \mathrm{~nm})$ und umgekehrt konnte nicht festgestellt werden.

Tartrazin und Rosanilin wurden wegen weit auseinander liegender Absorptionsmaxima als Farbstoffe gewählt (s. Abb. 1). Die in der Elektrophorese-Technik üblichen Farbstoffe Pouceau S und Amidoschwarz 10 B scheiden wegen der Lage der Absorptionskurven (13) aus.

Abbildung 1 zeigt, daß die Absorptionsmaxima von Tartrazin und Rosanilin genügend weit auseinanderliegen, um eine einwandfreie Messung zu garantieren. Eine wesentliche Beeinflussung der Proteinmessung durch den Kohlenhydrat-Farbstoff, der noch im Absorptionsmaximum von Tartrazin eine geringe Extinktion aufweist, die im Vergleich zur Extinkuion im $\mathrm{Ab}$ sorptionsmaximum von Tartrazin nur $14,6 \%$ beträgt, kann ausgeschlossen werden.

Bei Vergleich der spezifischen Färbungen auf parallel gelaufenen Streifen wären Versetzungen der Gipfelpunkte gegeneinander, wie sie die Abbildungen 2-5 zeigen, nicht mit Sicherheit auszumachen. Im Unterschied zu diesen Verfahren können mit der hier beschriebenen Methode Proteine und Kohlenhydrate auf einem Streifen beurteilt werden. Dies gewährleistet in jedem Fall, ohne daß man den Risiken bestimmter Techniken des Auftragens von Serum und der Inkonstanz des Spannungsfeldes ausgesetzt ist, eine exakte Aussage über die Zugehörigkeit der Kohlenhydrat- zu den Proteinfraktionen. Voraussetzung hierfür ist allerdings das einwandfreie und reproduzierbare Verhalten von Papiervorschub und Transportvorrichtung des Densitogrammzusatzes. Fehler lassen sich hier aber leicht erkennen, da dann bei Drehung der Glasplatte um $180^{\circ}$ die Markierungsstriche am Anfang und Ende des Elektropherogrammes nicht zueinander passen. 
Die genaue Kenntnis über die Zugehörigkeit von Glykoprotein-Fraktionen zu bestimmten Proteinen war uns besonders wichtig für unseren Beitrag zur „Differentialdiagnose“ M. WALdenström/Plasmocytom. Die Zahl der untersuchten Seren von Patienten mit M. WALDENSTRöm (4) und Plasmocytom (7) ist leider zu klein, um gesicherte Aussagen über das unterschiedliche Verhalten der Glykoproteine zu machen. Wie auch in einem von Wunderly und Piller (6) mitgeteilten Diagramm deutlich sichtbar ist, fiel uns bei den M. WALDENSTRÖMSeren der hohe, schmale und nur auf den $\boldsymbol{\gamma}$-GlobulinBereich beschränkte Kohlenhydrat-Gipfel auf. Im Gegensatz hierzu konnten die Glykoproteine je nach Art der immunologischen Differenzierung das Plasmocytom in verschiedene Globulin-Bereiche lokalisiert werden. Sie sind nach Form und Höhe des Ausschlages deutlich von den Diagrammen bei M. Waldenström unterschiedlich.

Dieses Verhalten ist Ausdruck der unterschiedlichen $\mathrm{Zu}-$ sammensetzung der Glykoproteine bei Paraproteinämien.
So wie die unterschiedlichen Globuline verschiedene Kohlenhydrat-Komponenten besitzen, durch die sie zusätzlich charakterisiert sind, ist auch den Paraproteinen eine bestimmte Kohlenhydrat-Komponente zugehörig. Bei einer ganzen Reihe akuter auch chronisch stationärer Krankheitsfälle sind die Veränderungen im Verhalten der Kohlenhydrate untersucht worden (SEIBERT und Mitarbeiter (11), E. KöIw und Mitarbeiter (5), Hartmann und Mitarbeiter (12), Wunderly und Mitarbeiter (6) und INFANTE und Mitarbeiter (8). Vergleichende Untersuchungen des Kohlenhydrat-Anteiles klinisch wichtiger Paraproteinämien stehen noch aus.

Es erscheint uns daher sinnvoll, diesen Eigentümlichkeiten des Verhalten der Glykoproteine mehr Beachtung zu schenken und anhand eines größeren Krankengutes zi überprüfen, weil eine Bestätigung unserer Vermutung die Differentialdiagnose der Paraproteinämien vereinfachen könnte.

\section{Literatur}

1. Mörner, K. A., Zbl. Physiol. 7, 581 (1893). - 2. Rimington, C., Biochem. J. 25, 1062 (1931). - 3. Sörensen, M. und G. Haugaard, Biochem. Z. 360, 247 (1933). - 4. Tiselius, A., Biochem. J. 31, 1464 (1931). - 5. Körw, E. und A. Grönvall, Scand. J. clin. Lab. Invest. T, 153 (1955). - 6. WUNDERLY, CH. und S. Piller, Klin. Wschr. 32, 425 (1954). - 7. Busse, V. und H.-J. Dulce, diese Z. 7, 486 (1969). - 8. Infante, F. und S. Girod-Jurllard, Clin. chim. Acta, Amsterdam 11, 424 (1965). -
9. Bötriger, L. E. und L. A. Carlson, Clin. chim. Acta, Amsterdam 5, 812 (1960). - 10. Scheiffarth, F., G. Berg und H. Görz, Papierelektrophorese in Klinik und Praxis, S. 39; Urban \& Schwarzenberg, München-Berlin (1962). - 11. Sexpert, F. B., M. V. Setbert, J. Atno und H. Campbel, J. clin. Invest. 26, 90 (1947). - 12. HartmanN, L., S. FilitTi-Wurmser, N. LelièvreArdaillou und P. Borvin, Sang 31, 491 (1960). - 13. Busse, V., diese Z. 6, 273 (1968).
Dr. V. Busse

1000 Berlin 19

Spandauer Damm 130 